\title{
Fourier series of finite product of Bernoulli and ordered Bell functions
}

\author{
Taekyun Kim ${ }^{a, b}$, Dae San Kimc, Dmitry V. Dolgy ${ }^{d}$, Jongkyum Kwon ${ }^{\mathrm{e}, *}$ \\ ${ }^{a}$ Department of Mathematics, College of Science, Tianjin Polytechnic University, Tianjin 300160, China. \\ ${ }^{b}$ Department of Mathematics, Kwangwoon University, Seoul, 139-701, Republic of Korea. \\ ${ }^{c}$ Department of Mathematics, Sogang University, Seoul, 121-742, Republic of Korea. \\ ${ }^{d}$ Hanrimwon, Kwangwoon University, Seoul, 139-701, Republic of Korea.
}

${ }^{e}$ Department of Mathematics Education and ERI, Gyeongsang National University, Jinju, Gyeongsangnamdo, 52828, Republic of Korea.

Communicated by K. Cieplinski

\begin{abstract}
In this paper, we consider three types of functions given by products of Bernoulli and ordered Bell functions and derive their Fourier series expansions. In addition, we will express each of them in terms of Bernoulli functions.
\end{abstract}

Keywords: Fourier series, Bernoulli functions, ordered Bell functions.

2010 MSC: $11 \mathrm{~B} 83,42 \mathrm{~A} 16$.

(C) 2018 All rights reserved.

\section{Introduction}

Let $B_{m}(x)$ denote the Bernoulli polynomials given by the generating function

$$
\frac{t}{e^{t}-1} e^{x t}=\sum_{m=0}^{\infty} B_{m}(x) \frac{t^{m}}{m !}, \quad(\text { see }[6,9,10,15,18]) .
$$

For $x=0, B_{m}=B_{m}(0)$ are called Bernoulli numbers. The Bernoulli polynomials satisfy the following properties.

$$
\frac{d}{d x} B_{m}(x)=m B_{m-1}(x),(m \geqslant 1), B_{m}(1)=B_{m}+\delta_{1, m},(m \geqslant 0) .
$$

For any real number $x$, we let

$$
\langle x\rangle=x-\lfloor x\rfloor \in[1,0)
$$

denote the fractional part of $x$. We also need the following facts about Bernoulli functions $B_{m}(\langle x\rangle)$ :

\footnotetext{
*Corresponding author

Email addresses: tkkim@kw.ac.kr (Taekyun Kim), dskim@sogang.ac.kr (Dae San Kim), dvdolgy@gmail.com (Dmitry V. Dolgy), mathkjk26@gnu.ac.kr (Jongkyum Kwon)
}

doi: $10.22436 /$ jnsa.011.04.07

Received: 2017-05-31 Revised: 2017-12-08 Accepted: 2017-12-26 
(a) for $m \geqslant 2, B_{m}(\langle x\rangle)=-m ! \sum_{\substack{n=-\infty \\ n \neq 0}}^{\infty} \frac{e^{2 \pi i n x}}{(2 \pi i n)^{m}}$;

(b) for $m=1,-\sum_{\substack{n=-\infty \\ n \neq 0}}^{\infty} \frac{e^{2 \pi i n x}}{2 \pi i n}= \begin{cases}B_{1}(\langle x\rangle), & \text { for } x \notin \mathbb{Z}, \\ 0, & \text { for } x \in \mathbb{Z} .\end{cases}$

The ordered Bell polynomials $b_{m}(x)$ are introduced as a natural companion to ordered Bell numbers $b_{m}=b_{m}(0)$ and defined by the generating function

$$
\frac{1}{2-t} e^{x t}=\sum_{m=0}^{\infty} b_{m}(x) \frac{t^{m}}{m !}, \quad(\text { see }[8]) .
$$

The first few ordered Bell polynomials are as follows:

$$
\begin{aligned}
& b_{0}(x)=1, b_{1}(x)=x+1, b_{2}(x)=x^{2}+2 x+3 \\
& b_{3}(x)=x^{3}+3 x^{2}+9 x+13, b_{4}(x)=x^{4}+4 x^{3}+18 x^{2}+52 x+75 \\
& b_{5}(x)=x^{5}+5 x^{4}+30 x^{3}+130 x^{2}+375 x+541
\end{aligned}
$$

The ordered Bell numbers have been studied in many counting problems in enumerative combinatorics and number theory, the first appearance of which goes back to as early as 1859. The ordered Bell polynomials are monic polynomials with integral coefficients, as we can see from

$$
b_{0}(x)=1, b_{m}(x)=x^{m}+\sum_{l=0}^{m-1}\left(\begin{array}{c}
m \\
l
\end{array}\right) b_{l}(x),(m \geqslant 1) .
$$

Also, the ordered Bell numbers are positive integers, as we can note from

$$
b_{m}=\sum_{n=0}^{m} n ! S_{2}(m, n)=\sum_{n=0}^{\infty} \frac{n^{m}}{2^{n+1}},(m \geqslant 0) .
$$

From (1.1), we can easily deduce

$$
\begin{aligned}
\frac{d}{d x} b_{m}(x) & =m b_{m-1}(x),(m \geqslant 1), \quad-b_{m}(1)+2 b_{m}=\delta_{m, 0},(m \geqslant 0), \\
\int_{0}^{1} b_{m}(x) d x & =\frac{1}{m+1}\left(b_{m+1}(1)-b_{m+1}\right)=\frac{1}{m+1} b_{m+1} .
\end{aligned}
$$

Throughout this paper, we will assume that $r$ and $s$ are nonnegative integers with $r+s \geqslant 1$. Here we will consider three types of sums of finite products of Bernoulli and ordered Bell functions $\alpha_{m}(\langle x\rangle), \beta_{m}(\langle x\rangle)$, and $\gamma_{m}(\langle x\rangle)$, and derive the Fourier series expansions of them. In addition, we will express each of them in terms of Bernoulli functions.

(1) $\alpha_{m}(\langle x\rangle)=\sum_{i_{1}+\cdots+i_{r}+j_{1}+\cdots+j_{s}=m} B_{i_{1}}(\langle x\rangle) \cdots B_{i_{r}}(\langle x\rangle) b_{j_{1}}(\langle x\rangle) \cdots b_{j_{s}}(\langle x\rangle), \quad(m \geqslant 1)$;

(2) $\beta_{m}(\langle x\rangle)=\sum_{i_{1}+\cdots+i_{r}+j_{1}+\cdots+j_{s}=m} \frac{1}{i_{1} ! \cdots i_{r} ! j_{1} ! \cdots j_{s} !} B_{i_{1}}(\langle x\rangle) \cdots B_{i_{r}}(\langle x\rangle) \times b_{j_{1}}(\langle x\rangle) \cdots b_{j_{s}}(\langle x\rangle), \quad(m \geqslant 1)$;

(3) $\gamma_{m}(\langle x\rangle)=\sum_{i_{1}+\cdots+i_{r}+j_{1}+\cdots+j_{s}=m} \frac{1}{i_{1} \cdots i_{r} j_{1} \cdots j_{s}} B_{i_{1}}(\langle x\rangle) \cdots B_{i_{r}}(\langle x\rangle) \times b_{j_{1}}(\langle x\rangle) \cdots b_{j_{s}}(\langle x\rangle),(m \geqslant r+s)$.

Here the sums for (1) and (2) run over all nonnegative integers $i_{1}, \cdots, i_{r}, j_{1}, \cdots, j_{s}$ with $i_{1}+\cdots+i_{r}+j_{1}+$ $\cdots+j_{s}=m$, and the sum for (3) runs over all positive integers $i_{1}, \cdots, i_{r}, j_{1}, \cdots, j_{s}$ with $i_{1}+\cdots+i_{r}+j_{1}+$ $\cdots+j_{s}=m$.

For elementary facts about Fourier analysis, the reader may refer to any book (for example, see [1, 16, 18]). As to $\alpha_{m}(\langle x\rangle)$, we note that the polynomial identity (1.2) follows immediately from Theorems 2.1 and 2.2, which is in turn derived from the Fourier series expansion of $\alpha_{m}(\langle x\rangle)$.

$$
\sum_{i_{1}+\cdots+i_{r}+j_{1}+\cdots+j_{s}=m} B_{i_{1}}(x) \cdots B_{i_{r}}(x) b_{j_{1}}(x) \cdots b_{j_{s}}(x)=\frac{1}{m+r+s} \sum_{j=0}^{m}\left(\begin{array}{c}
m+r+s \\
j
\end{array}\right) \Delta_{m-j+1} B_{j}(x),
$$


where, for each positive integer $l$,

$$
\begin{aligned}
\Delta_{l}= & \sum_{\substack{0 \leqslant a \leqslant r \\
0 \leqslant c \leqslant s \\
r-l \leqslant a \leqslant r}}\left(\begin{array}{l}
r \\
a
\end{array}\right)\left(\begin{array}{l}
s \\
c
\end{array}\right)(-1)^{s-c} 2^{c} \sum_{i_{1}+\cdots+i_{a}+j_{1}+\cdots+j_{c}=a+l-r} B_{i_{1}} \cdots B_{i_{a}} b_{j_{1}} \cdots b_{j_{c}} \\
& -\sum_{i_{1}+\cdots+i_{r}+j_{1}+\cdots+j_{s}=l} B_{i_{1}} \cdots B_{i_{r}} b_{j_{1}} \cdots b_{j_{s}} .
\end{aligned}
$$

The obvious polynomial identities can be derived also for $\beta_{m}(\langle x\rangle)$ from Theorems 3.1 and 3.2. It is noteworthy that from the Fourier series expansion of the function $\sum_{k=1}^{m-1} \frac{1}{k(m-k)} B_{k}(\langle x\rangle) B_{m-k}(\langle x\rangle)$ we can derive Faber-Pandharipande-Zagier identity (see $[4,5,9,10,17])$ and Miki's identity (see $[9,10$, 17]). Indeed, the next polynomial identity follows immediately from the Fourier series expansion of that function (see [13]):

$$
\begin{aligned}
\sum_{k=1}^{m-1} \frac{1}{k(m-k)} B_{k}(x) B_{m-k}(x)= & \frac{2}{m^{2}}\left(B_{m}+\frac{1}{2}\right)+\frac{2}{m} \sum_{k=1}^{m-2} \frac{1}{m-k}\left(\begin{array}{c}
m \\
k
\end{array}\right) B_{m-k} B_{k}(x) \\
& +\frac{2}{m} H_{m-1} B_{m}(x),
\end{aligned}
$$

where $m \geqslant 2$, and $H_{m}=\sum_{j=1}^{m} \frac{1}{j}$ are the harmonic numbers. Simple modification of (1.3) yields

$$
\begin{aligned}
\sum_{k=1}^{m-1} & \frac{1}{2 k(2 m-2 k)} B_{2 k}(x) B_{2 m-2 k}(x)+\frac{2}{2 m-1} B_{1}(x) B_{2 m-1}(x) \\
= & \frac{1}{m} \sum_{k=1}^{m} \frac{1}{2 k}\left(\begin{array}{c}
2 m \\
2 k
\end{array}\right) B_{2 k} B_{2 m-2 k}(x)+\frac{1}{m} H_{2 m-1} B_{2 m}(x) \\
& +\frac{2}{2 m-1} B_{1}(x) B_{2 m-1}, \quad(m \geqslant 2) .
\end{aligned}
$$

Letting $x=0$ in (1.4) gives a slightly different version of the well-known Miki's identity (see [17]):

$$
\sum_{k=1}^{m-1} \frac{1}{2 k(2 m-2 k)} B_{2 k} B_{2 m-2 k}=\frac{1}{m} \sum_{k=1}^{m} \frac{1}{2 k}\left(\begin{array}{c}
2 m \\
2 k
\end{array}\right) B_{2 k} B_{2 m-2 k}+\frac{1}{m} H_{2 m-1} B_{2 m}, \quad(m \geqslant 2) .
$$

Setting $x=\frac{1}{2}$ in (1.4) with $\bar{B}_{m}=\left(\frac{1-2^{m-1}}{2^{m-1}}\right) B_{m}=\left(2^{1-m}-1\right) B_{m}=B_{m}\left(\frac{1}{2}\right)$, we have

$$
\sum_{k=1}^{m-1} \frac{1}{2 k(2 m-2 k)} \bar{B}_{2 k} \bar{B}_{2 m-2 k}=\frac{1}{m} \sum_{k=1}^{m} \frac{1}{2 k}\left(\begin{array}{c}
2 m \\
2 k
\end{array}\right) B_{2 k} \bar{B}_{2 m-2 k}+\frac{1}{m} H_{2 m-1} \bar{B}_{2 m}, \quad(m \geqslant 2),
$$

which is the Faber-Pandharipande-Zagier identity (see [5]). Along the line of this paper, the Fourier series approach has been done for Bernoulli, Euler, Genocchi, poly-Bernoulli, poly-Genocchi, ordered Bell, r-derangement, derangement, Fubini, and two variable Fubini polynomials and also for their mixed types by the present authors and some other mathematicians. The reader may refer to the recent papers $[7,12-14]$ for a few related results.

\section{The function $\alpha_{\mathrm{m}}(\langle x\rangle)$}

Let $\alpha_{m}(x)=\sum_{\substack{j_{1}+\cdots+i_{r} \\ i_{1}+\cdots+j_{s}=m}} B_{i_{1}}(x) \cdots B_{i_{r}}(x) b_{j_{1}}(x) \cdots b_{j_{s}}(x),(m \geqslant 1)$, where the sum is over all nonnegative integers $i_{1}, \cdots, i_{r}, j_{1}, \cdots, j_{s}$ satisfying $i_{1}+\cdots+i_{r}+j_{1}+\cdots+j_{s}=m$.

Then we will consider the function

$$
\alpha_{m}(\langle x\rangle)=\sum_{i_{1}+\cdots+i_{r}+j_{1}+\cdots+j_{s}=m} B_{i_{1}}(\langle x\rangle) \cdots B_{i_{r}}(\langle x\rangle) b_{j_{1}}(\langle x\rangle) \cdots b_{j_{s}}(\langle x\rangle),
$$

which is a periodic function on $\mathbb{R}$ with period 1 . 
The Fourier series of $\alpha_{m}(\langle x\rangle)$ is

$$
\sum_{n=-\infty}^{\infty} A_{n}^{(m)} e^{2 \pi i n x}
$$

where

$$
A_{n}^{(m)}=\int_{0}^{1} \alpha_{m}(\langle x\rangle) e^{-2 \pi i n x} d x=\int_{0}^{1} \alpha_{m}(x) e^{-2 \pi i n x} d x .
$$

To continue our discussion, we need to observe the following:

$$
\begin{aligned}
\alpha_{m}^{\prime}(x)= & \sum_{i_{1}+\cdots+i_{r}+j_{1}+\cdots+i_{s}=m} i_{i_{1} \geqslant 1} B_{i_{1}-1}(x) B_{i_{2}}(x) \cdots B_{i_{r}}(x) b_{j_{1}}(x) \cdots b_{j_{s}}(x) \\
& +\cdots+\sum_{i_{1}+\cdots+i_{r}+j_{1}+\cdots+i_{s}=m} B_{i_{1}}(x) \cdots B_{i_{r}-1}(x) i_{r} B_{i_{r}-1}(x) b_{j_{1}}(x) \cdots b_{j_{s}}(x) \\
& +\sum_{i_{1}+\cdots+i_{r}+j_{1}+\cdots+i_{s}=m} B_{i_{1}}(x) \cdots B_{i_{r}}(x) j_{1} b_{j_{1}-1}(x) b_{j_{2}}(x) \cdots b_{j_{s}}(x) \\
& +\cdots+\sum_{i_{1}+\cdots+i_{r}+j_{1}+\cdots+i_{s}=m} B_{i_{1}}(x) \cdots B_{i_{r}}(x) b_{j_{1}}(x) \cdots b_{j_{s}-1}(x) j_{s} b_{j_{s}-1}(x) \\
= & \sum_{i_{1}+\cdots+i_{r}+j_{1}+\cdots j_{s}=m-1}\left(i_{1}+1\right) B_{i_{1}}(x) \cdots B_{i_{r}}(x) b_{j_{1}}(x) \cdots b_{j_{s}}(x) \\
& +\cdots+\sum_{i_{1}+\cdots+i_{r}+j_{1}+\cdots j_{s}=m-1}\left(i_{r}+1\right) B_{i_{1}}(x) \cdots B_{i_{r}}(x) b_{j_{1}}(x) \cdots b_{j_{s}}(x) \\
& +\sum_{i_{1}+\cdots+i_{r}+j_{1}+\cdots j_{s}=m-1}\left(j_{1}+1\right) B_{i_{1}}(x) \cdots B_{i_{r}}(x) b_{j_{1}}(x) \cdots b_{j_{s}}(x) \\
& +\cdots+\sum_{i_{1}+\cdots+i_{r}+j_{1}+\cdots j_{s}=m-1}\left(j_{s}+1\right) B_{i_{1}}(x) \cdots B_{i_{r}}(x) b_{j_{1}}(x) \cdots b_{j_{s}}(x) \\
= & (m+r+s-1) \alpha_{m-1}(x) .
\end{aligned}
$$

From (2.1), we immediately obtain

$$
\left(\frac{\alpha_{m+1}(x)}{m+r+s}\right)^{\prime}=\alpha_{m}(x)
$$

and

$$
\int_{0}^{1} \alpha_{m}(x) d x=\frac{1}{m+r+s}\left(\alpha_{m+1}(1)-\alpha_{m+1}(0)\right) .
$$

For $m \geqslant 1$, we let

$$
\begin{aligned}
\Delta_{\mathfrak{m}} & =\alpha_{\mathfrak{m}}(1)-\alpha_{\mathfrak{m}}(0) \\
& =\sum_{i_{1}+\cdots+i_{r}+j_{1}+\cdots j_{s}=m}\left(B_{i_{1}}(1) \cdots B_{i_{r}}(1) b_{j_{1}}(1) \cdots b_{j_{s}}(1)-B_{i_{1}} \cdots B_{i_{r}} b_{j_{1}} \cdots b_{j_{s}}\right) \\
& =\sum_{i_{1}+\cdots+i_{r}+j_{1}+\cdots j_{s}=m}\left(B_{i_{1}}+\delta_{1, i_{1}}\right) \cdots\left(B_{i_{r}}+\delta_{1, i_{r}}\right)\left(2 b_{j_{1}}-\delta_{j_{1}, 0}\right) \cdots\left(2 b_{j_{s}}-\delta_{j_{s}, 0}\right) \\
& -\sum_{i_{1}+\cdots+i_{r}+j_{1}+\cdots j_{s}=m} B_{i_{1}} \cdots B_{i_{r}} b_{j_{1}} \cdots b_{j_{s}}
\end{aligned}
$$




$$
\begin{aligned}
&= \sum_{\substack{0 \leqslant a \leqslant r \\
0 \leqslant c \leqslant s \\
r-m \leqslant a \leqslant r}}\left(\begin{array}{l}
r \\
a
\end{array}\right)\left(\begin{array}{l}
s \\
c
\end{array}\right)(-1)^{s-c} 2^{c} \sum_{i_{1}+\cdots+i_{a}+j_{1}+\cdots j_{c}=a+m-r} B_{i_{1}} \cdots B_{i_{a}} b_{j_{1}} \cdots b_{j_{c}} \\
&-\sum_{i_{1}+\cdots+i_{r}+j_{1}+\cdots j_{s}=m} B_{i_{1}} \cdots B_{i_{r}} b_{j_{1}} \cdots b_{j_{s}} .
\end{aligned}
$$

We note here that the sum over all $i_{1}+\cdots+i_{r}+j_{1}+\cdots j_{s}=m$ of any term with a of $B_{i_{e}}, r-a$ of $\delta_{1, i_{f}}$ $(1 \leqslant e, f \leqslant r), c$ of $2 b_{j_{u}}$, and $s-c$ of $-\delta_{j_{v}, 0}(1 \leqslant u, v \leqslant s)$ all give the same sum

$$
\begin{gathered}
\sum_{i_{1}+\cdots+i_{r}+j_{1}+\cdots+j_{s}=m} B_{i_{1}} \cdots B_{i_{a}} \delta_{1, i_{a}+1} \cdots \delta_{1, i_{r}}\left(2 b_{j_{1}}\right) \cdots\left(2 b_{j_{c}}\right)\left(-\delta_{j_{c}, 1}, 0\right) \cdots\left(-\delta_{j_{s}, 0}\right) \\
=\sum_{i_{1}+\cdots+i_{a}+j_{1}+\cdots+j_{c}=a+m-r}(-1)^{s-c} 2^{c} B_{i_{1}} \cdots B_{i_{a}} b_{j_{1}} \cdots b_{j_{c}}
\end{gathered}
$$

which is not an empty sum as long as $a+m-r \geqslant 0$.

We now see from (2.2) and (2.3) that

$$
\alpha_{\mathrm{m}}(0)=\alpha_{\mathrm{m}}(1) \Longleftrightarrow \Delta_{\mathrm{m}}=0, \quad \text { and } \quad \int_{0}^{1} \alpha_{\mathrm{m}}(\mathrm{x}) \mathrm{d} x=\frac{1}{\mathrm{~m}+\mathrm{r}+\mathrm{s}} \Delta_{\mathrm{m}+1} .
$$

We now would like to determine the Fourier coefficients $A_{n}^{(m)}$.

Case $1: n \neq 0$.

$$
\begin{aligned}
A_{n}^{(m)} & =\int_{0}^{1} \alpha_{m}(x) e^{-2 \pi i n x} d x \\
& =-\frac{1}{2 \pi i n}\left[\alpha_{m}(x) e^{-2 \pi i n x}\right]_{0}^{1}+\frac{1}{2 \pi i n} \int_{0}^{1} \alpha_{m}^{\prime}(x) e^{-2 \pi i n x} d x \\
& =-\frac{1}{2 \pi i n}\left(\alpha_{m}(1)-\alpha_{m}(0)\right)+\frac{m+r+s-1}{2 \pi i n} \int_{0}^{1} \alpha_{m-1}(x) e^{-2 \pi i n x} d x \\
& =\frac{m+r+s-1}{2 \pi i n} A_{n}^{(m-1)}-\frac{1}{2 \pi i n} \Delta m,
\end{aligned}
$$

from which by induction we can deduce

$$
A_{n}^{(m)}=-\sum_{j=1}^{m} \frac{(m+r+s-1)_{j-1}}{(2 \pi i n)^{j}} \Delta_{m-j+1}=-\frac{1}{m+r+s} \sum_{j=1}^{m} \frac{(m+r+s)_{j}}{(2 \pi i n)^{j}} \Delta_{m-j+1} .
$$

Case 2: $\mathfrak{n}=0$.

$$
A_{0}^{(m)}=\int_{0}^{1} \alpha_{m}(x) d x=\frac{1}{m+r+s} \Delta_{m+1}
$$

$\alpha_{m}(\langle x\rangle),(m \geqslant 1)$ is piecewise $C^{\infty}$. In addition, $\alpha_{m}(\langle x\rangle)$ is continuous for those positive integers with $\Delta_{\mathrm{m}}=0$, and discontinuous with jump discontinuities at integers for those positive integers with $\Delta_{\mathrm{m}} \neq 0$.

Assume first that $\Delta_{m}=0$ for a positive integer $m$. Then $\alpha_{m}(0)=\alpha_{m}(1)$. Hence $\alpha_{m}(\langle x\rangle)$ is piecewise $C^{\infty}$ and continuous. Hence the Fourier series of $\alpha_{m}(\langle x\rangle)$ converges uniformly to $\alpha_{m}(\langle x\rangle)$, and

$$
\begin{aligned}
\alpha_{m}(\langle x\rangle) & =\frac{1}{m+r+s} \Delta_{m+1}+\sum_{\substack{n=-\infty \\
n \neq 0}}^{\infty}\left(-\frac{1}{m+r+s} \sum_{j=1}^{m} \frac{(m+r+s)_{j}}{(2 \pi i n)^{j}} \Delta_{m-j+1}\right) e^{2 \pi i n x} \\
& =\frac{1}{m+r+s} \Delta_{m+1}+\frac{1}{m+r+s} \sum_{j=1}^{m}\left(\begin{array}{c}
m+r+s \\
j
\end{array}\right) \Delta_{m-j+1} \times\left(-j ! \sum_{\substack{n=-\infty \\
n \neq 0}}^{\infty} \frac{e^{2 \pi i n x}}{(2 \pi i n)^{j}}\right)
\end{aligned}
$$




$$
\begin{aligned}
= & \frac{1}{m+r+s} \Delta_{m+1}+\frac{1}{m+r+s} \sum_{j=2}^{\infty}\left(\begin{array}{c}
m+r+s \\
j
\end{array}\right) \Delta_{m-j+1} \\
& \times B_{j}(\langle x\rangle)+\Delta_{m} \times \begin{cases}B_{1}(\langle x\rangle), & \text { for } x \notin \mathbb{Z}, \\
0, & \text { for } x \in \mathbb{Z} .\end{cases}
\end{aligned}
$$

Now, we can state our first result.

Theorem 2.1. For each positive integer l, we let

$$
\begin{aligned}
\Delta_{l}= & \sum_{\substack{0 \leqslant a \leqslant r \\
0 \leqslant c \leqslant s \\
r-l \leqslant a \leqslant r}}\left(\begin{array}{l}
r \\
a
\end{array}\right)\left(\begin{array}{l}
s \\
c
\end{array}\right)(-1)^{s-c} 2^{c} \sum_{i_{1}+\cdots+i_{a}+j_{1}+\cdots+j_{c}=a+l-r} B_{i_{1}} \cdots B_{i_{a}} b_{j_{1}} \cdots b_{j_{c}} \\
& -\sum_{\substack{i_{1}+\cdots+i_{r}+j_{1}+\cdots+j_{s}=l \\
i_{1}}} B_{i_{1}} \cdots B_{i_{r}} b_{j_{1}} \cdots b_{j_{s}} .
\end{aligned}
$$

Assume that $\Delta_{\mathrm{m}}=0$, for a positive integer $\mathrm{m}$. Then we have the following:

(a)

$$
\sum_{i_{1}+\cdots+i_{r}+j_{1}+\cdots+j_{s}=m} B_{i_{1}}(\langle x\rangle) \cdots B_{i_{r}}\left(\langle x\rangle b_{j_{1}}(\langle x\rangle) \cdots b_{j_{s}}(\langle x\rangle)\right.
$$

has the Fourier series expansion

$$
\begin{gathered}
\sum_{i_{1}+\cdots+i_{r}+j_{1}+\cdots+j_{s}=m} B_{i_{1}}(\langle x\rangle) \cdots B_{i_{r}}(\langle x\rangle) b_{j_{1}}(\langle x\rangle) \cdots b_{j_{s}}(\langle x\rangle) \\
=\frac{1}{m+r+s} \Delta_{m+1}+\sum_{\substack{n=-\infty \\
n \neq 0}}^{\infty}\left(-\frac{1}{m+r+s} \sum_{j=1}^{m} \frac{(m+r+s)_{j}}{(2 \pi i n)^{j}} \Delta_{m-j+1}\right) e^{2 \pi i n x}
\end{gathered}
$$

for all $x \in \mathbb{R}$, where the convergence is uniform.

(b)

$$
\begin{aligned}
& \sum_{i_{1}+\cdots+i_{r}+j_{1}+\cdots+j_{s}=m} B_{i_{1}}(\langle x\rangle) \cdots B_{i_{r}}(\langle x\rangle) b_{j_{1}}(\langle x\rangle) \cdots b_{j_{s}}(\langle x\rangle) \\
&=\frac{1}{m+r+s} \Delta_{m+1}+\frac{1}{m+r+s} \sum_{j=2}^{m}\left(\begin{array}{c}
m+r+s \\
j
\end{array}\right) \Delta_{m-j+1} B_{j}(\langle x\rangle)
\end{aligned}
$$

for all $x \in \mathbb{R}$, where $\mathrm{B}_{\mathbf{j}}(\langle x\rangle)$ is the Bernoulli function.

Assume next that $\Delta_{m} \neq 0$ for a positive integer $m$. Then $\alpha_{m}(0) \neq \alpha_{m}(1)$. Hence $\alpha_{m}(\langle x\rangle)$ is piecewise $C^{\infty}$ and discontinuities at integers. The Fourier series of $\alpha_{\mathrm{m}}(\langle x\rangle)$ converges pointwise to $\alpha_{\mathrm{m}}(\langle x\rangle)$ for $x \notin \mathbb{Z}$, and converges to

$$
\frac{1}{2}\left(\alpha_{m}(0)+\alpha_{m}(1)\right)=\alpha_{m}(0)+\frac{1}{2} \Delta_{m}
$$

for $\in \mathbb{Z}$.

We can now state our second result. 
Theorem 2.2. For each positive integer $l$, we let

$$
\begin{aligned}
\Delta_{l}= & \sum_{\substack{0 \leqslant a \leqslant r \\
0 \leqslant c \leqslant s \\
r-l \leqslant a \leqslant r}}\left(\begin{array}{l}
r \\
a
\end{array}\right)\left(\begin{array}{l}
s \\
c
\end{array}\right)(-1)^{s-c} 2^{c} \sum_{i_{1}+\cdots+i_{a}+j_{1}+\cdots+j_{c}=a+l-r} B_{i_{1}} \cdots B_{i_{a}} b_{j_{1}} \cdots b_{j_{c}} \\
& -\sum_{\substack{i_{1}+\cdots+i_{r}+j_{1}+\cdots+j_{s}=l \\
i_{1}}} B_{i_{1}} \cdots B_{i_{r}} b_{j_{1}} \cdots b_{j_{s}} .
\end{aligned}
$$

Assume that $\Delta_{\mathrm{m}} \neq 0$ for a positive integer $\mathrm{m}$. Then we have the following:

(a)

$$
\begin{aligned}
& \frac{1}{m+r+s} \Delta_{m+1}+\sum_{\substack{n=-\infty \\
n \neq 0}}^{\infty}\left(-\frac{1}{m+r+s} \sum_{j=1}^{m} \frac{(m+r+s)_{j}}{(2 \pi i n)^{j}} \Delta_{m-j+1}\right) e^{2 \pi i n x} \\
& \quad= \begin{cases}\sum_{i_{1}+\cdots+i_{r}+j_{1}+\cdots+j_{s}=m} B_{i_{1}}(\langle x\rangle) \cdots B_{i_{r}}(\langle x\rangle) b_{j_{1}}(\langle x\rangle) \cdots b_{j_{s}}(\langle x\rangle), & \text { for } x \notin \mathbb{Z}, \\
\sum_{i_{1}+\cdots+i_{r}+j_{1}+\cdots+j_{s}=m} B_{i_{1}} \cdots B_{i_{r}} b_{j_{1}} \cdots b_{j_{s}}+\frac{1}{2} \Delta_{m}, & \text { for } x \in \mathbb{Z} .\end{cases}
\end{aligned}
$$

(b)

$$
\begin{aligned}
& \frac{1}{m+r+s} \sum_{j=0}^{m}\left(\begin{array}{c}
m+r+s \\
j
\end{array}\right) \Delta_{m-j+1} B_{j}(\langle x\rangle) \\
& =\sum_{i_{1}+\cdots+i_{r}+j_{1}+\cdots+j_{s}=m} B_{i_{1}}(\langle x\rangle) \cdots B_{i_{r}}(\langle x\rangle) b_{j_{1}}(\langle x\rangle) \cdots b_{j_{s}}(\langle x\rangle) \text { for } x \notin \mathbb{Z}, \\
& \frac{1}{m+r+s} \sum_{j=0}^{m}\left(\begin{array}{c}
m+r+s \\
j
\end{array}\right) \Delta_{m-j+1} B_{j}(\langle x\rangle) \\
& =\sum_{i_{1}+\cdots+i_{r}+j_{1}+\cdots+j_{s}=m} B_{i_{1}} \cdots B_{i_{r}} b_{j_{1}} \cdots b_{j_{s}}+\frac{1}{2} \Delta_{m} \text { for } x \in \mathbb{Z} .
\end{aligned}
$$

\section{The function $\beta_{m}(\langle x\rangle)$}

Let $\beta_{m}(x)=\sum_{\substack{j_{1}+\cdots+j_{s}=m \\ i_{1}+\cdots+i_{r}}} \frac{1}{i_{1} ! \cdots i_{r} ! j_{1} ! \cdots j_{s} !} B_{i_{1}}(x) \cdots B_{i_{r}}(x) b_{j_{1}}(x) \cdots b_{j_{s}}(x),(m \geqslant 1)$, where the sum runs over all nonnegative integers $i_{1}, \cdots, i_{r}, j_{1}, \cdots, j_{s}$ satisfying $i_{1}+\cdots+i_{r}+j_{1}+\cdots+j_{s}=m$.

Then we will consider the function

$$
\beta_{m}(\langle x\rangle)=\sum_{i_{1}+\cdots+i_{r}+j_{1}+\cdots+j_{s}=m} \frac{1}{i_{1} ! \cdots i_{r} ! j_{1} ! \cdots j_{s} !} B_{i_{1}}(\langle x\rangle) \cdots B_{i_{r}}(\langle x\rangle) b_{j_{1}}(\langle x\rangle) \cdots b_{j_{s}}(\langle x\rangle),
$$

which is a periodic function on $\mathbb{R}$ with period 1 .

The Fourier series of $\beta_{\mathfrak{m}}(\langle x\rangle)$ is

$$
\sum_{n=-\infty}^{\infty} B_{n}^{(m)} e^{2 \pi i n x}
$$

where

$$
B_{n}^{(m)}=\int_{0}^{1} \beta_{m}(\langle x\rangle) e^{-2 \pi i n x} d x=\int_{0}^{1} \beta_{m}(x) e^{-2 \pi i n x} d x .
$$

To continue our discussion, we need to notice the following. 


$$
\begin{aligned}
& \beta_{m}^{\prime}(x)=\sum_{\substack{i_{1}+\cdots+i_{r}+j_{1}+\cdots+i_{s}=m \\
i_{1} \geqslant 1}} \frac{1}{\left(i_{1}-1\right) ! i_{2} ! \cdots i_{r} ! j_{1} ! \cdots j_{s} !} B_{i_{1}-1}(x) B_{i_{2}}(x) \cdots B_{i_{r}}(x) \times b_{j_{1}}(x) \cdots b_{j_{s}}(x) \\
& +\cdots+\sum_{i_{1}+\cdots+i_{r}+j_{1}+\cdots+i_{s}=m} \frac{1}{i_{r} \geqslant 1} \underset{i_{1} ! \cdots i_{r-1} !\left(i_{r}-1\right) ! j_{1} ! \cdots j_{s} !}{ } B_{i_{1}}(x) \cdots B_{i_{r-1}}(x) B_{i_{r}-1}(x) \\
& \times b_{j_{1}}(x) \cdots b_{j_{s}}(x)+\sum_{i_{1}+\cdots+i_{r}+j_{1}+\cdots+i_{s}=m} \frac{1}{j_{1} \geqslant 1} \\
& \times b_{j_{1}-1}(x) b_{j_{2}}(x) \cdots b_{j_{s}}(x) \\
& +\cdots+\sum_{\substack{i_{1}+\cdots+i_{r}+j_{1}+\cdots+i_{s}=m \\
j_{s} \geqslant 1}} \frac{1}{i_{1} ! \cdots i_{r} ! j_{1} ! \cdots j_{s-1} !\left(j_{s}-1\right) !} B_{i_{1}}(x) \cdots B_{i_{r}}(x) \\
& \times b_{j_{1}}(x) \cdots b_{j_{s-1}}(x) b_{j_{s}-1}(x) \\
& =\sum_{i_{1}+\cdots+i_{r}+j_{1}+\cdots j_{s}=m-1} \frac{1}{i_{1} ! \cdots i_{r} ! j_{1} ! \cdots j_{s} !} B_{i_{1}}(x) \cdots B_{i_{r}}(x) b_{j_{1}}(x) \cdots b_{j_{s}}(x) \\
& +\cdots+\sum_{i_{1}+\cdots+i_{r}+j_{1}+\cdots j_{s}=m-1} \frac{1}{i_{1} ! \cdots i_{r} ! j_{1} ! \cdots j_{s} !} B_{i_{1}}(x) \cdots B_{i_{r}}(x) b_{j_{1}}(x) \cdots b_{j_{s}}(x) \\
& +\sum_{i_{1}+\cdots+i_{r}+j_{1}+\cdots j_{s}=m-1} \frac{1}{i_{1} ! \cdots i_{r} ! j_{1} ! \cdots j_{s} !} B_{i_{1}}(x) \cdots B_{i_{r}}(x) b_{j_{1}}(x) \cdots b_{j_{s}}(x) \\
& +\cdots+\sum_{i_{1}+\cdots+i_{r}+j_{1}+\cdots j_{s}=m-1} \frac{1}{i_{1} ! \cdots i_{r} ! j_{1} ! \cdots j_{s} !} B_{i_{1}}(x) \cdots B_{i_{r}}(x) b_{j_{1}}(x) \cdots b_{j_{s}}(x) \\
& =(\mathrm{r}+\mathrm{s}) \beta_{\mathrm{m}-1}(\mathrm{x}) \text {. }
\end{aligned}
$$

From (3.1), we get

$$
\left(\frac{\beta_{m+1}(x)}{r+s}\right)^{\prime}=\beta_{m}(x)
$$

and

$$
\int_{0}^{1} \beta_{\mathfrak{m}}(x) d x=\frac{1}{r+s}\left(\beta_{m+1}(1)-\beta_{m+1}(0)\right) .
$$

For $m \geqslant 1$, we let

$$
\begin{aligned}
\Omega_{m}= & \beta_{m}(1)-\beta_{m}(0) \\
= & \sum_{i_{1}+\cdots+i_{r}+j_{1}+\cdots j_{s}=m} \frac{1}{i_{1} ! \cdots i_{r} ! j_{1} ! \cdots j_{s} !}\left(B_{i_{1}}(1) \cdots B_{i_{r}}(1) b_{j_{1}}(1) \cdots b_{j_{s}}(1)-B_{i_{1}} \cdots B_{i_{r}} b_{j_{1}} \cdots b_{j_{s}}\right) \\
= & \sum_{i_{1}+\cdots+i_{r}+j_{1}+\cdots j_{s}=m} \frac{1}{i_{1} ! \cdots i_{r} ! j_{1} ! \cdots j_{s} !}\left(B_{i_{1}}+\delta_{1, i_{1}}\right) \cdots\left(B_{i_{r}}+\delta_{1, i_{r}}\right) \\
& \times\left(2 b_{j_{1}}-\delta_{j_{1}, 0}\right) \cdots\left(2 b_{j_{s}}-\delta_{j_{s}, 0}\right) \\
& -\sum_{\substack{i_{1}+\cdots+i_{r}+j_{1}+\cdots j_{s}=m \\
=}} \sum_{\substack{0 \leqslant a \leqslant r \\
0 \leqslant c \leqslant s}}\left(\begin{array}{l}
r \\
a
\end{array}\right)\left(\begin{array}{l}
s \\
c
\end{array}\right)(-1)^{s-c} 2^{c} \sum_{i_{1}+\cdots+i_{a}+j_{1}+\cdots j_{c}=a+m-r} \sum_{i_{r} ! j_{1} ! \cdots j_{s} !}^{c} B_{i_{1}} \cdots B_{i_{r}} b_{j_{1}} \cdots b_{j_{s}} \\
& \times B_{i_{1} \cdots i_{a} ! j_{1} ! \cdots j_{c} !} b_{j_{1}} \cdots b_{j_{c}}-\sum_{i_{1}+\cdots+i_{r}+j_{1}+\cdots j_{s}=m} \frac{1}{i_{1} ! \cdots i_{r} ! j_{1} ! \cdots j_{s} !} B_{i_{1}} \cdots B_{i_{r}} b_{j_{1}} \cdots b_{j_{s}} .
\end{aligned}
$$


Now, we see from (3.2) and (3.3) that

$$
\beta_{\mathfrak{m}}(0)=\beta_{\mathfrak{m}}(1) \Longleftrightarrow \Omega_{\mathfrak{m}}=0 \text { and } \quad \int_{0}^{1} \beta_{\mathfrak{m}}(x) \mathrm{d} x=\frac{1}{r+s} \Omega_{\mathrm{m}+1} .
$$

We now want to determine the Fourier coefficients $B_{n}^{(m)}$.

Case $1: n \neq 0$.

$$
\begin{aligned}
B_{n}^{(m)} & =\int_{0}^{1} \beta_{m}(x) e^{-2 \pi i n x} d x \\
& =-\frac{1}{2 \pi i n}\left[\beta_{m}(x) e^{-2 \pi i n x}\right]_{0}^{1}+\frac{1}{2 \pi i n} \int_{0}^{1} \beta_{m}^{\prime}(x) e^{-2 \pi i n x} d x \\
& =-\frac{1}{2 \pi i n}\left(\beta_{m}(1)-\beta_{m}(0)\right)+\frac{r+s}{2 \pi i n} \int_{0}^{1} \beta_{m-1}(x) e^{2 \pi i n x} d x \\
& =\frac{r+s}{2 \pi i n} B_{n}^{(m-1)}-\frac{1}{2 \pi i n} \Omega_{m}
\end{aligned}
$$

from which we can easily deduce that

$$
B_{n}^{(m)}=-\sum_{j=1}^{m} \frac{(r+s)^{j-1}}{(2 \pi i n)^{j}} \Omega_{m-j+1} .
$$

Case 2: $\mathrm{n}=0$.

$$
\mathrm{B}_{0}^{(\mathrm{m})}=\int_{0}^{1} \beta_{\mathrm{m}}(x) \mathrm{d} x=\frac{1}{\mathrm{r}+\mathrm{s}} \Omega_{\mathrm{m}+1} .
$$

$\beta_{m}(\langle x\rangle),(m \geqslant 1)$ is piecewise $C^{\infty}$. Moreover, $\beta_{m}(\langle x\rangle)$ is continuous for those positive integers $m$ with $\Omega_{m}=0$, and discontinuous with jump discontinuities at integers for those positive integers $m$ with $\Omega_{\mathrm{m}} \neq 0$.

Assume first that $\Omega_{m}=0$, for a positive integer $m$. Then $\beta_{m}(0)=\beta_{m}(1)$. Hence $\beta_{m}(\langle x\rangle)$ is piecewise $C^{\infty}$ and continuous. Thus the Fourier series of $\beta_{m}(\langle x\rangle)$ converges uniformly to $\beta_{m}(\langle x\rangle)$, and

$$
\begin{aligned}
\beta_{m}(\langle x\rangle) & =\frac{1}{r+s} \Omega_{m+1}+\sum_{\substack{n=-\infty \\
n \neq 0}}^{\infty}\left(-\sum_{j=1}^{m} \frac{(r+s)^{j-1}}{(2 \pi i n)^{j}} \Omega_{m-j+1}\right) e^{2 \pi i n x} \\
& =\frac{1}{r+s} \Omega_{m+1}+\sum_{j=1}^{m} \frac{(r+s)^{j-1}}{j !} \Omega_{m-j+1} \times\left(-j ! \sum_{\substack{n=-\infty \\
n \neq 0}}^{\infty} \frac{e^{2 \pi i n x}}{(2 \pi i n)^{j}}\right) \\
& =\frac{1}{r+s} \Omega_{m+1}+\sum_{j=2}^{m} \frac{(r+s)^{j-1}}{j !} \Omega_{m-j+1} B_{j}(\langle x\rangle)+\Omega_{m} \times \begin{cases}B_{1}(\langle x\rangle), & \text { for } x \notin \mathbb{Z}, \\
0, & \text { for } x \in \mathbb{Z} .\end{cases}
\end{aligned}
$$

We are now ready to state our first result.

Theorem 3.1. For each positive integer $l$, we let

$$
\begin{aligned}
\Omega_{l}= & \sum_{\substack{0 \leqslant a \leqslant r \\
0 \leqslant c \leqslant s \\
r-l \leqslant a \leqslant r}}\left(\begin{array}{l}
r \\
a
\end{array}\right)\left(\begin{array}{l}
s \\
c
\end{array}\right)(-1)^{s-c} 2^{c} \sum_{i_{1}+\cdots+i_{a}+j_{1}+\cdots+j_{c}=a+l-r} \frac{B_{i_{1}} \cdots B_{i_{a}} b_{j_{1}} \cdots b_{j_{c}}}{i_{1} ! \cdots i_{a} ! j_{1} ! \cdots j_{c} !} \\
& -\sum_{i_{1}+\cdots+i_{r}+j_{1}+\cdots+j_{s}=l} \frac{B_{i_{1}} \cdots B_{i_{r}} b_{j_{1}} \cdots b_{j_{s}}}{i_{1} ! \cdots i_{r} ! j_{1} ! \cdots j_{s} !} .
\end{aligned}
$$

Assume that $\Omega_{\mathrm{m}}=0$ for a positive integer $\mathrm{m}$. Then we have the following. 
(a)

$$
\sum_{i_{1}+\cdots+i_{r}+j_{1}+\cdots+j_{s}=m} \frac{1}{i_{1} ! \cdots i_{r} ! j_{1} ! \cdots j_{s} !} B_{i_{1}}(\langle x\rangle) \cdots B_{i_{r}}\left(\langle x\rangle b_{j_{1}}(\langle x\rangle) \cdots b_{j_{s}}(\langle x\rangle)\right.
$$

has the Fourier series expansion

$$
\begin{aligned}
& \sum_{i_{1}+\cdots+i_{r}+j_{1}+\cdots+j_{s}=m} \frac{1}{i_{1} ! \cdots i_{r} ! j_{1} ! \cdots j_{s} !} B_{i_{1}}(\langle x\rangle) \cdots B_{i_{r}}(\langle x\rangle) b_{j_{1}}(\langle x\rangle) \cdots b_{j_{s}}(\langle x\rangle) \\
= & \frac{1}{r+s} \Omega_{m+1}+\sum_{\substack{n=-\infty \\
n \neq 0}}^{\infty}\left(-\sum_{j=1}^{m} \frac{(r+s)^{j-1}}{(2 \pi i n)^{j}} \Omega_{m-j+1}\right) e^{2 \pi i n x}
\end{aligned}
$$

for all $x \in \mathbb{R}$, where the convergence is uniform.

(b)

$$
\begin{aligned}
& \sum_{\substack{j=0 \\
i_{1}+\cdots+i_{r}+j_{1}+\cdots+j_{s}=m}} \frac{1}{i_{1} ! \cdots i_{r} ! j_{1} ! \cdots j_{s} !} B_{i_{1}}(\langle x\rangle) \cdots B_{i_{r}}(\langle x\rangle) b_{j_{1}}(\langle x\rangle) \cdots b_{j_{s}}(\langle x\rangle) \\
=\sum_{\substack{j \neq 1 \\
j \neq 1}}^{(r+s)^{j-1}} & \Omega_{m-j+1} B_{j}(\langle x\rangle)
\end{aligned}
$$

for all $x \in \mathbb{R}$, where $\mathrm{B}_{\mathbf{j}}(\langle x\rangle)$ is the Bernoulli function.

Assume next that $\Omega_{m} \neq 0$, for a positive integer $m$. Then $\beta_{m}(0) \neq \beta_{m}(1)$. Hence $\beta_{m}(\langle x\rangle)$ is piecewise $C^{\infty}$, and discontinuities with jump discontinuities at integers. Thus the Fourier series of $\beta_{\mathrm{m}}(\langle x\rangle)$ converges pointwise to $\beta_{\mathrm{m}}(\langle x\rangle)$ for $x \notin \mathbb{Z}$, and converges to

$$
\frac{1}{2}\left(\beta_{\mathfrak{m}}(0)+\beta_{m}(1)\right)=\beta_{\mathfrak{m}}(0)+\frac{1}{2} \Omega_{\mathfrak{m}}
$$

for $\in \mathbb{Z}$.

Now, we are ready to state our second result.

Theorem 3.2. For each positive integer $l$, we let

$$
\begin{aligned}
\Omega_{l}= & \sum_{\substack{0 \leqslant a \leqslant r \\
0 \leqslant c \leqslant s \\
r-l \leqslant a \leqslant r}}\left(\begin{array}{l}
r \\
a
\end{array}\right)\left(\begin{array}{l}
s \\
c
\end{array}\right)(-1)^{s-c} 2^{c} \sum_{i_{1}+\cdots+i_{a}+j_{1}+\cdots+j_{c}=a+l-r} \frac{B_{i_{1}} \cdots B_{i_{a}} b_{j_{1}} \cdots b_{j_{c}}}{i_{1} ! \cdots i_{a} ! j_{1} ! \cdots j_{c} !} \\
& -\sum_{i_{1}+\cdots+i_{r}+j_{1}+\cdots+j_{s}=l} \frac{B_{i_{1}} \cdots B_{i_{r}} b_{j_{1}} \cdots b_{j_{s}}}{i_{1} ! \cdots i_{r} ! j_{1} ! \cdots j_{s} !} .
\end{aligned}
$$

Assume that $\Omega_{\mathrm{m}} \neq 0$, for a positive integer $\mathrm{m}$. Then we have the following:

(a)

$$
\begin{aligned}
\frac{1}{r+s} & \Omega_{m+1}+\sum_{\substack{n=-\infty \\
n \neq 0}}^{\infty}\left(-\sum_{j=1}^{m} \frac{(r+s)^{j-1}}{(2 \pi i n)^{j}} \Omega_{m-j+1}\right) e^{2 \pi i n x} \\
& = \begin{cases}\sum_{i_{1}+\cdots+i_{r}+j_{1}+\cdots+j_{s}=m} \frac{1}{i_{1} ! \cdots i_{r} ! j_{1} ! \cdots j_{s} !} B_{i_{1}}(\langle x\rangle) \cdots B_{i_{r}}(\langle x\rangle) b_{j_{1}}(\langle x\rangle) \cdots b_{j_{s}}(\langle x\rangle), & \text { for } x \notin \mathbb{Z}, \\
\sum_{i_{1}+\cdots+i_{r}+j_{1}+\cdots+j_{s}=m} \frac{B_{i_{1}} \cdots B_{i_{r}} b_{j_{1}} \cdots b_{j_{s}}}{i_{1} ! \cdots i_{r} ! j_{1} ! \cdots j_{s} !}+\frac{1}{2} \Omega_{m}, & \text { for } x \in \mathbb{Z} .\end{cases}
\end{aligned}
$$


(b)

$$
\begin{aligned}
& \sum_{j=0}^{m} \frac{(r+s)^{j-1}}{j !} \Omega_{m-j+1} B_{j}(\langle x\rangle) \\
& \quad=\sum_{\substack{j=0 \\
i_{1}+\cdots+i_{r}+j_{1}+\cdots+j_{s}=m}} \frac{1}{i_{1} ! \cdots i_{r} ! j_{1} ! \cdots j_{s} !} B_{i_{1}}(\langle x\rangle) \cdots B_{i_{r}}(\langle x\rangle) b_{j_{1}}(\langle x\rangle) \cdots b_{j_{s}}(\langle x\rangle) \text { for } x \notin \mathbb{Z}, \\
& \sum_{\substack{m \\
j}}^{m} \frac{(r+s)^{j-1}}{j m-j+1} B_{j}(\langle x\rangle)=\sum_{i_{1}+\cdots+i_{r}+j_{1}+\cdots+j_{s}=m} \frac{B_{i_{1}} \cdots B_{i_{r}} b_{j_{1}} \cdots b_{j_{s}}}{i_{1} ! \cdots i_{r} ! j_{1} ! \cdots j_{s} !}+\frac{1}{2} \Omega_{m} \text { for } x \in \mathbb{Z} .
\end{aligned}
$$

\section{The function $\gamma_{r, s, m}(\langle x\rangle)$}

Let $\gamma_{r, s, m}(x)=\sum_{\substack{i_{1}+\cdots+i_{r} \\+j_{1}+\cdots+j_{s}=m}} \frac{1}{i_{1} \cdots i_{r} j_{1} \cdots j_{s}} B_{i_{1}}(x) \cdots B_{i_{r}}(x) b_{j_{1}}(x) \cdots b_{j_{s}}(x),(m \geqslant r+s)$, where the sum is over all positive integers $i_{1}, \cdots, i_{r}, j_{1}, \cdots, j_{s}$ satisfying $i_{1}+\cdots+i_{r}+j_{1}+\cdots+j_{s}=m$.

Then we will consider the function

$$
\gamma_{r, s, m}(\langle x\rangle)=\sum_{i_{1}+\cdots+i_{r}+j_{1}+\cdots+j_{s}=m} \frac{1}{i_{1} \cdots i_{r} j_{1} \cdots j_{s}} B_{i_{1}}(\langle x\rangle) \cdots B_{i_{r}}(\langle x\rangle) b_{j_{1}}(\langle x\rangle) \cdots b_{j_{s}}(\langle x\rangle),
$$

which is a periodic function on $\mathbb{R}$ with period 1 .

The Fourier series of $\gamma_{r, s, m}(\langle x\rangle)$ is

$$
\sum_{n=-\infty}^{\infty} C_{n}^{(r, s, m)} e^{2 \pi i n x}
$$

where

$$
C_{n}^{(r, s, m)}=\int_{0}^{1} \gamma_{r, s, m}(\langle x\rangle) e^{-2 \pi i n x} d x=\int_{0}^{1} \gamma_{r, s, m}(x) e^{-2 \pi i n x} d x
$$

To proceed further, we need to observe the following.

$$
\begin{aligned}
\gamma_{r, s, m}^{\prime}(x)= & \sum_{i_{1}+\cdots+i_{r}+j_{1}+\cdots+j_{s}=m} \frac{1}{i_{2} \cdots i_{r} j_{1} \cdots j_{s}} B_{i_{1}-1}(x) B_{i_{2}}(x) \cdots B_{i_{r}}(x) b_{j_{1}}(x) \cdots b_{j_{s}}(x) \\
& +\cdots+\sum_{i_{1}+\cdots+i_{r}+j_{1}+\cdots+j_{s}=m} \frac{1}{i_{1} \cdots i_{r-1} j_{1} \cdots j_{s}} B_{i_{1}}(x) \cdots B_{i_{r-1}}(x) B_{i_{r}-1}(x) b_{j_{1}}(x) \cdots b_{j_{s}}(x) \\
& +\sum_{i_{1}+\cdots+i_{r}+j_{1}+\cdots+j_{s}=m} \frac{1}{i_{1} \cdots i_{r} j_{2} \cdots j_{s}} B_{i_{1}}(x) \cdots B_{i_{r}}(x) b_{j_{1}-1}(x) b_{j_{2}}(x) \cdots b_{j_{s}}(x) \\
& +\cdots+\sum_{i_{1}+\cdots+i_{r}+j_{1}+\cdots+j_{s}=m} \frac{1}{i_{1} \cdots i_{r} j_{1} \cdots j_{s}-1} B_{i_{1}}(x) \cdots B_{i_{r}}(x) b_{j_{1}}(x) \cdots b_{j_{s-1}}(x) b_{j_{s}-1}(x) \\
= & \sum_{i_{2}+\cdots+i_{r}+j_{1}+\cdots+j_{s}=m-1} \frac{1}{i_{2} \cdots i_{r} j_{1} \cdots j_{s}} B_{i_{2}}(x) \cdots B_{i_{r}}(x) b_{j_{1}}(x) \cdots b_{j_{s}}(x) \\
& +\sum_{i_{1}+\cdots+i_{r}+j_{1}+\cdots+j_{s}=m-1} \frac{1}{i_{2} \cdots i_{r} j_{1} \cdots j_{s}} B_{i_{1}}(x) \cdots B_{i_{r}}(x) b_{j_{1}}(x) \cdots b_{j_{s}}(x) \\
& +\cdots+\sum_{i_{1}+\cdots+i_{r-1}+j_{1}+\cdots+j_{s}=m-1} \frac{1}{i_{1} \cdots i_{r}-1 j_{1} \cdots j_{s}} B_{i_{1}}(x) \cdots B_{i_{r-1}}(x) b_{j_{1}}(x) \cdots b_{j_{s}}(x)
\end{aligned}
$$




$$
\begin{aligned}
& +\sum_{i_{1}+\cdots+i_{r}+j_{1}+\cdots+j_{s}=m-1} \frac{1}{i_{1} \cdots i_{r-1} j_{1} \cdots j_{s}} B_{i_{1}}(x) \cdots B_{i_{r}}(x) b_{j_{1}}(x) \cdots b_{j_{s}}(x) \\
& +\sum_{i_{1}+\cdots+i_{r}+j_{2}+\cdots+j_{s}=m-1} \frac{1}{i_{1} \cdots i_{r} j_{2} \cdots j_{s}} B_{i_{1}}(x) \cdots B_{i_{r}}(x) b_{j_{2}}(x) \cdots b_{j_{s}}(x) \\
& +\sum_{i_{1}+\cdots+i_{r}+j_{1}+\cdots+j_{s}=m-1} \frac{1}{i_{1} \cdots i_{r} j_{2} \cdots j_{s}} B_{i_{1}}(x) \cdots B_{i_{r}}(x) b_{j_{1}}(x) \cdots b_{j_{s}}(x) \\
& +\cdots+\sum_{i_{1}+\cdots+i_{r}+j_{1}+\cdots+j_{s-1}=m-1} \frac{1}{i_{1} \cdots i_{r} j_{1} \cdots j_{s-1}} B_{i_{1}}(x) \cdots B_{i_{r}}(x) b_{j_{1}}(x) \cdots b_{j_{s}-1}(x) \\
& +\sum_{i_{1}+\cdots+i_{r}+j_{1}+\cdots+j_{s}=m-1} \frac{1}{i_{1} \cdots i_{r} j_{1} \cdots j_{s-1}} B_{i_{1}}(x) \cdots B_{i_{r}}(x) b_{j_{1}}(x) \cdots b_{j_{s}}(x) \\
& +\sum_{i_{1}+\cdots+i_{r}+j_{1}+\cdots+j_{s}=m-1}\left\{\frac{1}{i_{2} \cdots i_{r} j_{1} \cdots j_{s}}+\cdots+\frac{1}{i_{1} \cdots i_{r-1} j_{1} \cdots j_{s}}\right. \\
& \left.+\frac{1}{i_{1} \cdots i_{r} j_{2} \cdots j_{s}}+\cdots+\frac{1}{i_{1} \cdots i_{r} j_{1} \cdots j_{s-1}}\right\} B_{i_{1}}(x) \cdots B_{i_{r}}(x) b_{j_{1}}(x) \cdots b_{j_{s}}(x) \\
& =r \gamma_{r-1, s, m-1}(x)+s \gamma_{r, s-1, m-1}(x)+(m-1) \\
& \gamma_{r, s, m-1}(x) .
\end{aligned}
$$

Thus we obtain

$$
\gamma_{r, s, m}^{\prime}(x)=r \gamma_{r-1, s, m-1}(x)+s \gamma_{r, s-1, m-1}(x)+(m-1) \gamma_{r, s, m-1}(x),
$$

with $\gamma_{\mathrm{r}, \mathrm{s}, \mathrm{r}+\mathrm{s}-1}(\mathrm{x})=0$.

For $m \geqslant r+s$, we set

$$
\begin{aligned}
\Lambda_{r, s, m}= & \gamma_{r, s, m}(1)-\gamma_{r, s, m}(0) \\
= & \sum_{i_{1}+\cdots+i_{r}+j_{1}+\cdots+j_{s}=m} \frac{1}{i_{1} \cdots i_{r} j_{1} \cdots j_{s}}\left(B_{i_{1}}(1) \cdots B_{i_{r}}(1) b_{j_{1}}(1) \cdots b_{j_{s}}(1)-B_{i_{1}} \cdots B_{i_{r}} b_{j_{1}} \cdots b_{j_{s}}\right) \\
= & \sum_{i_{1}+\cdots+i_{r}+j_{1}+\cdots+j_{s}=m} \frac{1}{i_{1} \cdots i_{r} j_{1} \cdots j_{s}}\left(\left(B_{i_{1}}+\delta_{1, i_{1}}\right) \cdots\left(B_{i_{r}}+\delta_{1, i_{r}}\right)\right. \\
& \left.\times\left(2 b_{j_{1}}-\delta_{0, j_{1}}\right) \cdots\left(2 b_{j_{s}}-\delta_{0, j_{s}}\right)\right) \\
& -\sum_{i_{1}+\cdots+i_{r}+j_{1}+\cdots+j_{s}=m} \frac{1}{i_{1} \cdots i_{r} j_{1} \cdots j_{s}} B_{i_{1}} \cdots B_{i_{r}} b_{j_{1}} \cdots b_{j_{s}} \\
= & \sum_{a=0}^{r}\left(\begin{array}{l}
r \\
a
\end{array}\right) \sum_{i_{1}+\cdots+i_{a}+j_{1}+\cdots+j_{s}=a+m-r} \frac{1}{i_{1} \cdots i_{a} j_{1} \cdots j_{s}} B_{i_{1}} \cdots B_{i_{a}} b_{j_{1}} \cdots b_{j_{s}} \\
& -\sum_{i_{1}+\cdots+i_{r}+j_{1}+\cdots+j_{s}=m} \frac{1}{i_{1} \cdots i_{r} j_{1} \cdots j_{s}} B_{i_{1}} \cdots B_{i_{r}} b_{j_{1}} \cdots b_{j_{s}} .
\end{aligned}
$$

Our next task is to determine

$$
a_{r, s, m}=\int_{0}^{1} \gamma_{r, s, m}(x) d x
$$

From (4.1), we immediately obtain

$$
a_{r, s, m}=-\frac{r}{m} a_{r-1, s, m}(x)-\frac{s}{m} a_{r, s-1, m}(x)+\frac{1}{m} \Lambda_{r, s, m+1} .
$$


We showed in [2] that

$$
a_{r, 0, m}=\int_{0}^{1} \gamma_{r, 0, m}(x) d x=\sum_{j=1}^{r} \frac{(-1)^{j-1}(r)_{j-1}}{m^{j}} \Lambda_{r-j+1,0, m+1}, \quad(r \geqslant 1) .
$$

We also derived in [3] that

$$
a_{0, s, m}=\int_{0}^{1} \gamma_{0, s, m}(x) d x=\sum_{j=1}^{s} \frac{(-1)^{j-1}(s)_{j-1}}{m^{j}} \Lambda_{0, s-j+1, m+1}, \quad(s \geqslant 1) .
$$

It is now clear that (4.2) together with (4.3) and (4.4) determines $a_{r, s, m}$ for all $r, s, m$ with $m \geqslant r+s \geqslant 1$. Also, we observe that

$$
\gamma_{\mathrm{r}, \mathrm{s}, \mathrm{m}}(0)=\gamma_{\mathrm{r}, \mathrm{s}, \mathrm{m}}(1) \Leftrightarrow \Lambda_{\mathrm{r}, \mathrm{s}, \mathrm{m}}=0 .
$$

Now, we would like to determine the Fourier coefficients $C_{n}^{(r, s, m)}$.

Case $1: n \neq 0$.

$$
\begin{aligned}
& C_{n}^{(r, s, m)}=\int_{0}^{1} \gamma_{r, s, m}(x) e^{-2 \pi i n x} d x \\
& =-\frac{1}{2 \pi i n}\left[\gamma_{r, s, m}(x) e^{-2 \pi i n x}\right]_{0}^{1}+\frac{1}{2 \pi i n} \int_{0}^{1} \gamma_{r, s, m}^{\prime}(x) e^{-2 \pi i n x} d x \\
& =-\frac{1}{2 \pi i n}\left(\gamma_{r, s, m}(1)-\gamma_{r, s, m}(0)\right)+\frac{1}{2 \pi i n} \int_{0}^{1}\left\{r \gamma_{r-1, s, m-1}(x)+s \gamma_{r, s-1, m-1}(x)\right. \\
& \left.+(m-1) \gamma_{r, s, m-1}(x)\right\} e^{-2 \pi i n x} d x \\
& =-\frac{1}{2 \pi i n} \Lambda_{r, s, m}+\frac{1}{2 \pi i n}\left(r C_{n}^{(r-1, s, m-1)}+s C_{n}^{(r, s-1, m-1)}+(m-1) C_{n}^{(r, s, m-1)}\right) \\
& =\frac{m-1}{2 \pi i n} C_{n}^{(r, s, m-1)}+\frac{r}{2 \pi i n} C_{n}^{(r-1, s, m-1)}+\frac{s}{2 \pi i n} C_{n}^{(r, s-1, m-1)}-\frac{1}{2 \pi i n} \Lambda_{r, s, m} \\
& =\frac{m-1}{2 \pi i n}\left(\frac{m-2}{2 \pi i n} C_{n}^{(r, s, m-2)}+\frac{r}{2 \pi i n} C_{n}^{(r-1, s, m-2)}+\frac{s}{2 \pi i n} C_{n}^{(r, s-1, m-2)}\right. \\
& \left.-\frac{1}{2 \pi i n} \Lambda_{r, s, m-1}\right)+\frac{r}{2 \pi i n} C_{n}^{(r-1, s, m-1)}+\frac{s}{2 \pi i n} C_{n}^{(r, s-1, m-1)}-\frac{1}{2 \pi i n} \Lambda_{r, s, m} \\
& =\frac{(m-1)_{2}}{(2 \pi i n)^{2}} C_{n}^{(r, s, m-2)}+\sum_{j=1}^{2} \frac{r(m-1)_{j-1}}{(2 \pi i n)^{j}} C_{n}^{(r-1, s, m-j)} \\
& +\sum_{j=1}^{2} \frac{s(m-1)_{j-1}}{(2 \pi i n)^{j}} C_{n}^{(r, s-1, m-j)}-\sum_{j=1}^{2} \frac{(m-1)_{j-1}}{(2 \pi i n)^{j}} \Lambda_{r, s, m-j+1} \\
& =\cdots=\frac{(m-1)_{m-(r+s)}}{(2 \pi i n)^{m-(r+s)}} C_{n}^{(r, s, r+s)}+\sum_{j=1}^{m-(r+s)} \frac{r(m-1)_{j-1}}{(2 \pi i n)^{j}} C_{n}^{(r-1, s, m-j)} \\
& +\sum_{j=1}^{m-(r+s)} \frac{s(m-1)_{j-1}}{(2 \pi i n)^{j}} C_{n}^{(r, s-1, m-j)}-\sum_{j=1}^{m-(r+s)} \frac{(m-1)_{j-1}}{(2 \pi i n)^{j}} \Lambda_{r, s, m-j+1} \\
& =\sum_{j=1}^{m-(r+s)+1} \frac{r(m-1)_{j-1}}{(2 \pi i n)^{j}} C_{n}^{(r-1, s, m-j)}+\sum_{j=1}^{m-(r+s)+1} \frac{s(m-1)_{j-1}}{(2 \pi i n)^{j}} C_{n}^{(r, s-1, m-j)} \\
& -\sum_{j=1}^{m-(r+s)+1} \frac{(m-1)_{j-1}}{(2 \pi i n)^{j}} \Lambda_{r, s, m-j+1} .
\end{aligned}
$$


Here we note that

$$
\begin{aligned}
C_{n}^{(r, s, r+s)}= & \int_{0}^{1}\left(x-\frac{1}{2}\right)^{r}(x+1)^{s} e^{-2 \pi i n x} d x \\
= & -\frac{1}{2 \pi i n}\left(\left(\frac{1}{2}\right)^{r} 2^{s}-\left(-\frac{1}{2}\right)^{r}\right)+\frac{r}{2 \pi i n} \int_{0}^{1}\left(x-\frac{1}{2}\right)^{r-1}(x+1)^{s} e^{-2 \pi i n x} d x \\
& +\frac{s}{2 \pi i n} \int_{0}^{1}\left(x-\frac{1}{2}\right)^{r}(x+1)^{s-1} e^{-2 \pi i n x} d x \\
= & -\frac{1}{2 \pi i n} \Lambda_{r, s, r+s}+\frac{r}{2 \pi i n} C_{n}^{(r-1, s, r+s-1)}+\frac{s}{2 \pi i n} C_{n}^{(r, s-1, r+s-1)} .
\end{aligned}
$$

Thus we have shown that

$$
\begin{aligned}
C_{n}^{(r, s, m)}= & \sum_{j=1}^{m-(r+s)+1} \frac{r(m-1)_{j-1}}{(2 \pi i n)^{j}} C_{n}^{(r-1, s, m-j)}+\sum_{j=1}^{m-(r+s)+1} \frac{s(m-1)_{j-1}}{(2 \pi i n)^{j}} C_{n}^{(r, s-1, m-j)} \\
& -\sum_{j=1}^{m-(r+s)+1} \frac{(m-1)_{j-1}}{(2 \pi i n)^{j}} \Lambda_{r, s, m-j+1} .
\end{aligned}
$$

Also, from [2] and [3] we recall that

$$
\begin{aligned}
& C_{n}^{(r, 0, m)}=\sum_{j=1}^{m-r+1} \frac{r(m-1)_{j-1}}{(2 \pi i n)^{j}} C_{n}^{(r-1,0, m-j)}-\sum_{j=1}^{m-r+1} \frac{(m-1)_{j-1}}{(2 \pi i n)^{j}} \Lambda_{r, 0, m-j+1}, \quad(r \geqslant 2), \\
& C_{n}^{(1,0, m)}=-\frac{(m-1) !}{(2 \pi i n)^{m}} \\
& C_{n}^{(0, s, m)}=\sum_{j=1}^{m-s+1} \frac{s(m-1)_{j-1}}{(2 \pi i n)^{j}} C_{n}^{(0, s-1, m-j)}-\sum_{j=1}^{m-s+1} \frac{(m-1)_{j-1}}{(2 \pi i n)^{j}} \Lambda_{0, s, m-j+1}, \quad(s \geqslant 2), \\
& C_{n}^{(0,1, m)}=-\frac{1}{m} \sum_{j=1}^{m} \frac{(m)_{j-1}}{(2 \pi i n)^{j}} b_{m-j+1} .
\end{aligned}
$$

We see that $C_{n}^{(r, s, m)}(n \neq 0)$ can be determined for all $m \geqslant r+s \geqslant 1$ from (4.5)-(4.9). Case $2: n=0$.

$$
\mathrm{C}_{0}^{(\mathrm{r}, \mathrm{s}, \mathrm{m})}=\int_{0}^{1} \gamma_{\mathrm{r}, \mathrm{s}, \mathrm{m}}(\mathrm{x}) \mathrm{d} \mathrm{x}
$$

can be determined for all $m \geqslant r+s \geqslant 1$ from (4.2)-(4.4).

$\gamma_{r, s, m}(\langle x\rangle),(m \geqslant r+s \geqslant 1)$ is piecewise $C^{\infty}$. Furthermore, $\gamma_{r, s, m}(\langle x\rangle)$ is continuous for those integers $r, s, m$ with $\Lambda_{r, s, m}=0$ and discontinuous with jump discontinuities at integers for those integers $r, s, m$ with $\wedge_{r, s, m} \neq 0$.

Assume first that $\Lambda_{r, s, m}=0$ for some integers $r, s, m$ with $m \geqslant r+s \geqslant 1$. Then $\gamma_{r, s, m}(0)=\gamma_{r, s, m}(1)$. Thus $\gamma_{r, s, m}(\langle x\rangle)$ is piecewise $C^{\infty}$ and continuous. Thus the Fourier series of $\gamma_{r, s, m}(\langle x\rangle)$ converges uniformly to $\gamma_{r, s, m}(\langle x\rangle)$, and

$$
\gamma_{r, s, m}(\langle x\rangle)=C_{0}^{(r, s, m)}+\sum_{n=-\infty, n \neq 0}^{\infty} C_{n}^{(r, s, m)} e^{2 \pi i n x},
$$

where $C_{0}^{(r, s, m)}$ are determined by (4.2)-(4.4) and $C_{n}^{(r, s, m)}(n \neq 0)$ by (4.5)-(4.9).

We are now ready to state our first result. 
Theorem 4.1. For all integers $r, s, l$ with $l \geqslant r+s \geqslant 1$, we let

$$
\begin{aligned}
\Lambda_{r, s, l}= & \sum_{a=0}^{r}\left(\begin{array}{l}
r \\
a
\end{array}\right) \sum_{i_{1}+\cdots+i_{a}+j_{1}+\cdots+j_{s}=a+l-r} \frac{2^{s}}{i_{1} \cdots i_{a} j_{1} \cdots j_{s}} B_{i_{1}} \cdots B_{i_{a}} b_{j_{1}} \cdots b_{j_{s}} \\
& -\sum_{i_{1}+\cdots+i_{r}+j_{1}+\cdots+j_{s}=l} \frac{1}{i_{1} \cdots i_{r} j_{1} \cdots j_{s}} B_{i_{1}} \cdots B_{i_{r}} b_{j_{1}} \cdots b_{j_{s}} .
\end{aligned}
$$

Assume that $\Lambda_{r, s, m}=0$ for some integers $r, s, m$ with $m \geqslant r+s \geqslant 1$. Then we have the following.

$$
\sum_{i_{1}+\cdots+i_{r}+j_{1}+\cdots+j_{s}=m} \frac{1}{i_{1} \cdots i_{r} j_{1} \cdots j_{s}} B_{i_{1}}(\langle x\rangle) \cdots B_{i_{r}}(\langle x\rangle) \times b_{j_{1}}(\langle x\rangle) \cdots b_{j_{s}}(\langle x\rangle)
$$

has the Fourier series expansion

$$
\begin{aligned}
& \sum_{i_{1}+\cdots+i_{r}+j_{1}+\cdots+j_{s}=m} \frac{1}{i_{1} \cdots i_{r} j_{1} \cdots j_{s}} B_{i_{1}}(\langle x\rangle) \cdots B_{i_{r}}(\langle x\rangle) \times b_{j_{1}}(\langle x\rangle) \cdots b_{j_{s}}(\langle x\rangle) \\
=C_{0}^{(r, s, m)}+ & \sum_{n=-\infty, n \neq 0}^{\infty} C_{n}^{(r, s, m)} e^{2 \pi i n x}
\end{aligned}
$$

where $\mathrm{C}_{0}^{(\mathrm{r}, \mathrm{s}, \mathrm{m})}$ are determined by (4.2)-(4.4) and $\mathrm{C}_{\mathrm{n}}^{(\mathrm{r}, \mathrm{s}, \mathrm{m})}(\mathrm{n} \neq 0)$ by (4.5)-(4.9). Here the convergence is uniform.

Next, assume that $\Lambda_{r, s, m} \neq 0$, for some integers $r, s, m$ with $m \geqslant r+s \geqslant 1$. Then $\gamma_{r, s, m}(0) \neq \gamma_{r, s, m}(1)$. Hence $\gamma_{r, s, m}(\langle x\rangle)$ is piecewise $C^{\infty}$ and discontinuous with jump discontinuities at integers. Then the Fourier series of $\gamma_{r, s, m}(\langle x\rangle)$ converges pointwise to $\gamma_{r, s, m}(\langle x\rangle)$, for $x \notin \mathbb{Z}$, and converges to

$$
\frac{1}{2}\left(\gamma_{r, s, m}(0)+\gamma_{r, s, m}(1)\right)=\gamma_{r, s, m}(0)+\frac{1}{2} \Lambda_{r, s, m}
$$

for $x \in \mathbb{Z}$.

Next, we can state our second result.

Theorem 4.2. For all integers $r, s, l$ with $l \geqslant r+s \geqslant 1$, we let

$$
\begin{aligned}
\Lambda_{r, s, l}= & \sum_{a=0}^{r}\left(\begin{array}{l}
r \\
a
\end{array}\right) \sum_{i_{1}+\cdots+i_{a}+j_{1}+\cdots+j_{s}=a+l-r} \frac{2^{s}}{i_{1} \cdots i_{a} j_{1} \cdots j_{s}} B_{i_{1}} \cdots B_{i_{a}} b_{j_{1}} \cdots b_{j_{s}} \\
& -\sum_{i_{1}+\cdots+i_{r}+j_{1}+\cdots+j_{s}=l} \frac{1}{i_{1} \cdots i_{r} j_{1} \cdots j_{s}} B_{i_{1}} \cdots B_{i_{r}} b_{j_{1}} \cdots b_{j_{s}} .
\end{aligned}
$$

Assume that $\Lambda_{r, s, m} \neq 0$ for some integers $r, s, m$ with $m \geqslant r+s \geqslant 1$. Then we have the following.

$$
\begin{aligned}
& C_{0}^{(r, s, m)}+\sum_{n=-\infty, n \neq 0}^{\infty} C_{n}^{(r, s, m)} e^{2 \pi i n x} \\
& =\left\{\begin{array}{l}
\sum_{i_{1}+\cdots+i_{r}+j_{1}+\cdots+j_{s}=m} \frac{1}{i_{1} \cdots i_{r} j_{1} \cdots j_{s}} B_{i_{1}}(\langle x\rangle) \cdots B_{i_{r}}(\langle x\rangle) \times b_{j_{1}}(\langle x\rangle) \cdots b_{j_{s}}(\langle x\rangle) \text { for } x \notin \mathbb{Z}, \\
\sum_{i_{1}+\cdots+i_{r}+j_{1}+\cdots+j_{s}=m} \frac{1}{i_{1} \cdots i_{r} j_{1} \cdots j_{s}} B_{i_{1}} \cdots B_{i_{r}} b_{j_{1}} \cdots b_{j_{s}}+\frac{1}{2} \Lambda_{r, s, m} \text { for } x \in \mathbb{Z},
\end{array}\right.
\end{aligned}
$$

where $\mathrm{C}_{\mathrm{n}}^{(\mathrm{r}, \mathrm{s}, \mathrm{m})}(\mathrm{n} \neq 0)$ are determined by (4.5)-(4.9) and $\mathrm{C}_{0}^{(\mathrm{r}, \mathrm{s}, \mathrm{m})}$ by (4.2)-(4.4).

\section{Acknowledgment}

We would like to thank the referee for his detailed suggestions which improved the manuscript greatly. 


\section{References}

[1] M. Abramowitz, I. A. Stegun, Handbook of Mathematical Functions with formulas, graphs, and mathematical tables. National Bureau of Standards Applied Mathematics Series, Government Printing Office, Washington, D.C., (1964). 1

[2] R. P. Agarwal, D. S. Kim, T. Kim, J. Kwon, Sums of finite products of Bernoulli functions, Adv. Difference Equ., 2017 (2017), 15 pages. 4,4

[3] D. V. Dolgy, D. S. Kim, T. Kim, T. Mansour, Sums of finite products of ordered Bell functions, preprint. 4, 4

[4] G. V. Dunne, C. Schubert, Bernoulli number identities from quantum field theory and topological string theory, Commun. Number Theory Phys., 7 (2013), 225-249. 1

[5] C. Faber, R. Pandharipande, Hodge integrals and Gromov-Witten theory, Invent. Math., 139 (2000), 173-199. 1, 1

[6] S. Gaboury, R. Tremblay, B.-J. Fugére, Some explicit formulas for certain new classes of Bernoulli, Euler and Genocchi polynomials, Proc. Jangjeon Math. Soc., 17 (2014), 115-123. 1

[7] G.-W. Jang, T. Kim, D. S. Kim, T. Mansour, Fourier series of functions related to Bernoulli polynomials, Adv. Stud. Contemp. Math., 27 (2017), 49-62. 1

[8] T. Kim, Some identities for the Bernoulli, the Euler and Genocchi numbers and polynomials, Adv. Stud. Contemp. Math., 20 (2010), 23-28. 1.1

[9] D. S. Kim, T. Kim, Bernoulli basis and the product of several Bernoulli polynomials, Int. J. Math. Math. Sci., 2012 (2012), 12 pages. 1,1

[10] D. S. Kim, T. Kim, A note on higher-order Bernoulli polynomials, J. Inequal. Appl., 2013 (2013), 9 pages. 1, 1

[11] D. S. Kim, T. Kim, Identities arising from higher-order Daehee polynomial bases, Open Math., 13 (2015), $196-208$.

[12] T. Kim, D. S. Kim, D. V. Dolgy, J.-W. Park, Fourier series of sums of products of ordered Bell and poly-Bernoulli functions, J. Inequal. Appl., 2017 (2017), 17pages. 1

[13] T. Kim, D. S. Kim, G.-W. Jang, J. Kwon, Fourier series of sums of products of Genocchi functions and their applications, J. Nonlinear Sci. Appl., 10 (2017), 1683-1694. 1

[14] T. Kim, D. S. Kim, S.-H. Rim, D. Dolgy, Fourier series of higher-order Bernoulli functions and their applications, J. Inequal. Appl., 2017 (2017), 8 pages. 1

[15] H. Liu, W. Wang, Some identities on the Bernoulli, Euler and Genocchi polynomials via power sums and alternate power sums, Discrete Math., 309 (2009), 3346-3363. 1

[16] J. E. Marsden, Elementary classical analysis, W. H. Freeman and Company, San Francisco, (1974). 1

[17] H. Miki, A relation between Bernoulli numbers, J. Number Theory, 10 (1978), 297-302. 1, 1

[18] H. M. Srivastava, Some generalizations and basic (or q-) extensions of the Bernoulli, Euler and Genocchi polynomials, Appl. Math. Inf. Sci., 5 (2011), 390-444. 1, 1 